\title{
Iron-Modulated 3D CoNiP Vertical Nanoarrays: An Exploratory Binder-Free Bifunctional Electrocatalyst for Efficient Overall Water Splitting
}

Manigandan Ramadoss ${ }^{\mathrm{a}, \mathrm{b} *}$, Yuanfu Chen ${ }^{\mathrm{a}, \mathrm{c} *}, \mathrm{Xin}_{\mathrm{Chen}}{ }^{\mathrm{a}}, \mathrm{Zhe} \mathrm{Su}^{\mathrm{a}}$, Marimuthu Karpuraranjith ${ }^{\mathrm{a}}$, Dongxu Yanga ${ }^{\mathrm{a}}$, Manzoor Ahmad Pandit ${ }^{\mathrm{b}}$, and Krishnamurthi Muralidharan ${ }^{\mathrm{b} *}$

${ }^{a}$ School of Electronic Science and Engineering, and State Key Laboratory of Electronic Thin Films and Integrated Devices, University of Electronic Science and Technology of China, Chengdu 610054, PR China

${ }^{b}$ School of Chemistry, University of Hyderabad, Hyderabad 500046, India

'School of Science, and Institute of Oxygen Supply, Tibet University, Lhasa 850000, PR China

*Email: yfchen@uestc.edu.cn (Y.F.Chen),rmaniunom@gmail.com (R. Manigandan),murali@uoyd.ac.in (K. Muralidharan) 
Table. S1: Summarized values of Fe/CoNiP@NF performance with other recently reported transition-metal-based materials as multifunctional electrocatalysts.

\begin{tabular}{|c|c|c|c|c|c|}
\hline Materials & n mV@10 mA/cm² & $\begin{array}{l}\text { Tafel } \\
\text { slope } \\
\text { (mV/ } \\
\text { dec) } \\
\end{array}$ & $\begin{array}{l}\text { (i-t) } \\
\text { (h) }\end{array}$ & Electrolyte & Ref. \\
\hline $\mathrm{Co}_{2} \mathrm{P} / \mathrm{Co}$-foil & $\begin{array}{l}319 \text { (OER) } \\
157 \text { (HER) } \\
480 \text { (OWS) }\end{array}$ & $\begin{array}{r}79 \\
59 \\
-\end{array}$ & $\begin{array}{l}12 \\
12 \\
15\end{array}$ & $1.0 \mathrm{M} \mathrm{KOH}$ & 1 \\
\hline $\mathrm{CoP} / \mathrm{rGO}-400$ & $\begin{array}{l}105 \text { (HER) } \\
150 \text { (HER) } \\
340 \text { (OER) } \\
470 \text { (OWS) }\end{array}$ & $\begin{array}{c}50 \\
38 \\
66 \\
135 \\
\end{array}$ & $\begin{array}{l}22 \\
22 \\
22 \\
22 \\
\end{array}$ & $\begin{array}{c}0.5 \mathrm{M} \\
\mathrm{H}_{2} \mathrm{SO}_{4} \\
1.0 \mathrm{M} \mathrm{KOH}\end{array}$ & 2 \\
\hline $\mathrm{CoP} / \mathrm{CoP}_{2} / \mathrm{Al}_{2} \mathrm{O}_{3}$ & $\begin{array}{c}300 \text { OER } \\
181 \text { (HER) } \\
420 \text { (OWS) }\end{array}$ & $\begin{array}{l}63 \\
73\end{array}$ & $\begin{array}{l}24 \\
24 \\
24\end{array}$ & $1.0 \mathrm{M} \mathrm{KOH}$ & 3 \\
\hline $\mathrm{Ni}_{0.7} \mathrm{Fe}_{0.3} \mathrm{~S}_{2} @ \mathrm{NF}$ & $\begin{array}{l}198 \text { (OER) } \\
155 \text { (HER) } \\
395 \text { (OWS) }\end{array}$ & $\begin{array}{c}56 \\
109 \\
- \\
\end{array}$ & $\begin{array}{l}\sim 14 \\
\sim 14 \\
\sim 14 \\
\end{array}$ & $1.0 \mathrm{M} \mathrm{KOH}$ & 4 \\
\hline FCP-CN & $\begin{array}{c}80 \text { (HER) } \\
178 \text { (HER) } \\
323 \text { (OWS) }\end{array}$ & $\begin{array}{l}57 \\
71 \\
38\end{array}$ & $\begin{array}{l}22 \\
22 \\
22\end{array}$ & $\begin{array}{c}0.5 \mathrm{M} \\
\mathrm{H}_{2} \mathrm{SO}_{4} \\
1.0 \mathrm{M} \mathrm{KOH}\end{array}$ & 5 \\
\hline Co-P-B-5 & $\begin{array}{l}145 \text { (HER) } \\
290 \text { (OER) } \\
420 \text { (OWS) }\end{array}$ & $\begin{array}{l}38 \\
42 \\
-\end{array}$ & $\begin{array}{l}20 \\
20 \\
20\end{array}$ & $\begin{array}{l}1.0 \mathrm{M} \\
\mathrm{NaOH}\end{array}$ & 6 \\
\hline $\begin{array}{l}\mathrm{HP}-\mathrm{Co}_{3} \mathrm{O}_{4} \\
\text { arrays/NF }\end{array}$ & $\begin{array}{l}205 \text { (OER) } \\
118 \text { (HER) } \\
350 \text { (OWS) }\end{array}$ & $\begin{array}{c}65.3 \\
102.8 \\
-\end{array}$ & $\begin{array}{l}24 \\
24 \\
24\end{array}$ & $1.0 \mathrm{M} \mathrm{KOH}$ & 7 \\
\hline $\mathrm{NiFe} / \mathrm{Ni} / \mathrm{Ni}$ & $\begin{array}{c}132 \text { (HER) } \\
190 \text { (OER) } \\
730 \text { (n500) (OWS) }\end{array}$ & $\begin{array}{c}107.7 \\
108.1 \\
- \\
\end{array}$ & $\begin{array}{c}72 \\
72 \\
200 \\
\end{array}$ & $1.0 \mathrm{M} \mathrm{KOH}$ & 8 \\
\hline NiFe-MOF/NF & $\begin{array}{c}240 \text { (OER) } \\
134 \text { (HER) } \\
1.55 \mathrm{~V} \text { (OWS) }\end{array}$ & $\begin{array}{c}34 \\
- \\
256\end{array}$ & $\begin{array}{l}\sim 6 \\
\sim 6 \\
20 \\
\end{array}$ & $0.1 \mathrm{M} \mathrm{KOH}$ & 9 \\
\hline $\mathrm{NiFe} \mathrm{LDH} / \mathrm{NF}$ & $\begin{array}{l}240 \text { (OER) } \\
210 \text { (HER) }\end{array}$ & $\begin{array}{l}- \\
-\end{array}$ & $\begin{array}{l}- \\
-\end{array}$ & $1.0 \mathrm{M} \mathrm{KOH}$ & 10 \\
\hline$\left(\mathrm{Ni}_{0.33} \mathrm{Fe}_{0.67}\right)_{2} \mathrm{P}$ & $\begin{array}{l}214 \text { (n50) (HER) } \\
230 \text { (n50) (OER) } \\
260 \text { (n10) (OWS) }\end{array}$ & $\begin{array}{c}- \\
55.9 \\
- \\
\end{array}$ & $\begin{array}{l}12 \\
12 \\
12 \\
\end{array}$ & $1.0 \mathrm{M} \mathrm{KOH}$ & 11 \\
\hline Fe-CoNiP@NF & $\begin{array}{l}280 \text { (OER) } \\
110 \text { (HER) } \\
390 \text { (OWS) }\end{array}$ & $\begin{array}{c}91.9 \\
92.6 \\
-\end{array}$ & $\begin{array}{l}49 \\
60 \\
16\end{array}$ & $\begin{array}{l}1.0 \mathrm{M} \\
\mathrm{KOH}\end{array}$ & $\begin{array}{l}\text { This } \\
\text { work }\end{array}$ \\
\hline
\end{tabular}


Table. S2: Summarized LSV, Cdl, and EIS of samples

\begin{tabular}{|c|c|c|c|c|c|c|}
\hline \multirow[b]{2}{*}{ Materials } & \multicolumn{2}{|c|}{ HER } & \multicolumn{2}{|c|}{ OER } & \multirow{2}{*}{ 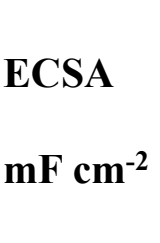 } & \multirow{2}{*}{$\begin{array}{l}\text { EIS } \\
\Omega\end{array}$} \\
\hline & $\begin{array}{l}\eta \quad @ \quad 10 \\
\text { mA } / \mathbf{c m}^{2}\end{array}$ & $\begin{array}{l}\eta @ 10 \\
\mathrm{~mA} / \mathrm{cm}^{2}\end{array}$ & $\begin{array}{l}\mathrm{n} @ 10 \\
\mathrm{~mA} / \mathrm{cm}^{2}\end{array}$ & $\begin{array}{l}\text { Tafel } \\
\text { (mV/dec) }\end{array}$ & & \\
\hline $\mathrm{Ni}_{\mathbf{x}} \mathrm{P}$ & $160 \mathrm{mV}$ & $114.9 \mathrm{mV}$ & $395 \mathrm{mV}$ & $132.9 \mathrm{mV}$ & 1.41 & 42.3 \\
\hline CoNiP & $135 \mathrm{mV}$ & $108.2 \mathrm{mV}$ & $310 \mathrm{mV}$ & $102.8 \mathrm{mV}$ & 17.9 & 14.4 \\
\hline Fe-CoNiP & $110 \mathrm{mV}$ & $92.6 \mathrm{mV}$ & $280 \mathrm{mV}$ & $91.9 \mathrm{mV}$ & 41.2 & 10.8 \\
\hline
\end{tabular}

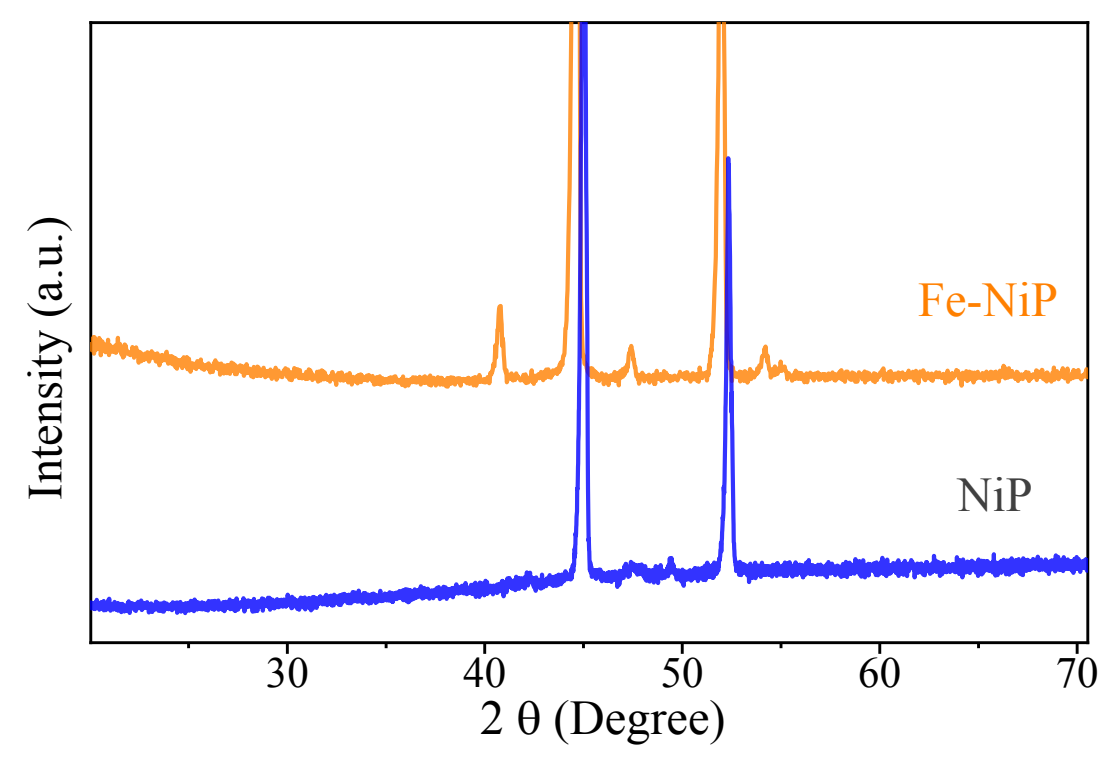

Fig. S1: The XRD patterns NiP and Fe-NiP electrocatalysts 


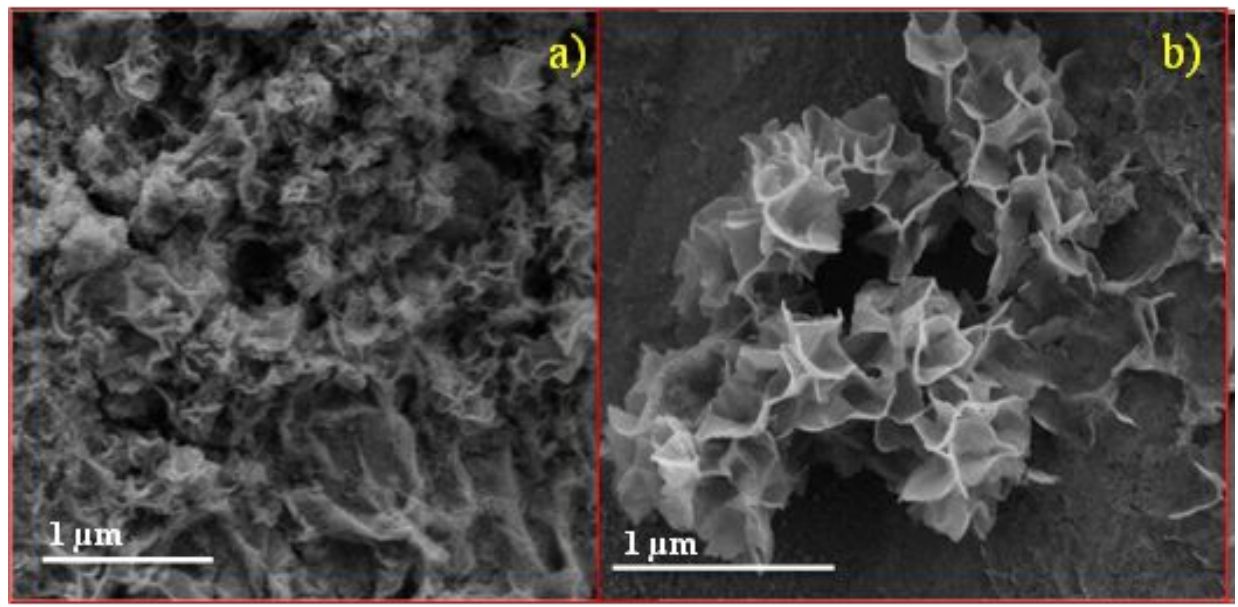

Fig. S2: The SEM images of a) NiP and b) Fe-NiP electrocatalysts.

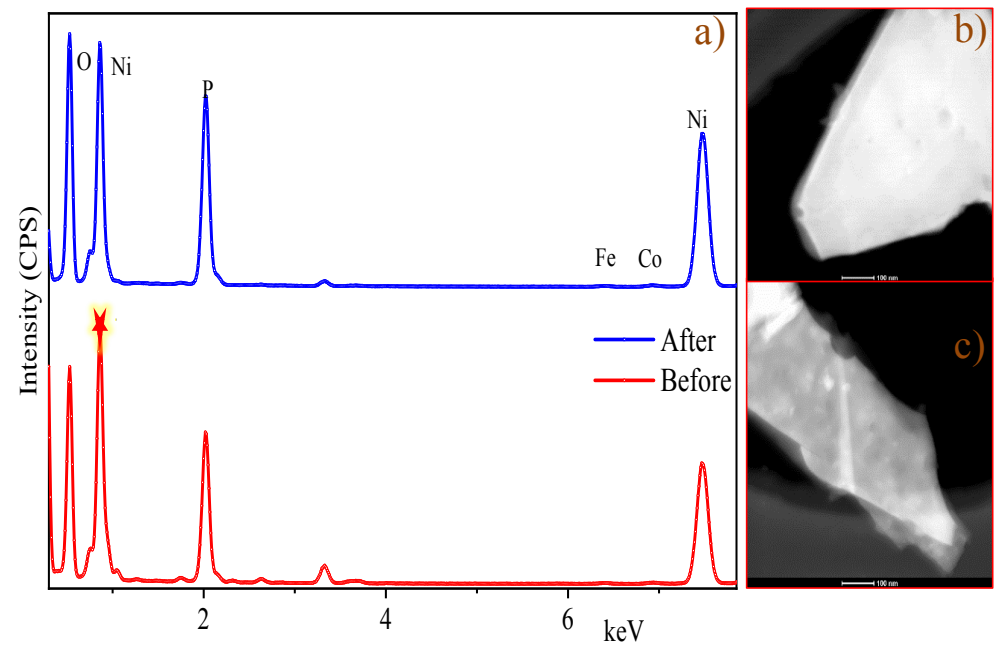

Fig. S3: a) EDX spectra of before and after b) HADFF images b) before, and c) after electrolysis of Fe-CoNiP@NF electrocatalyst.
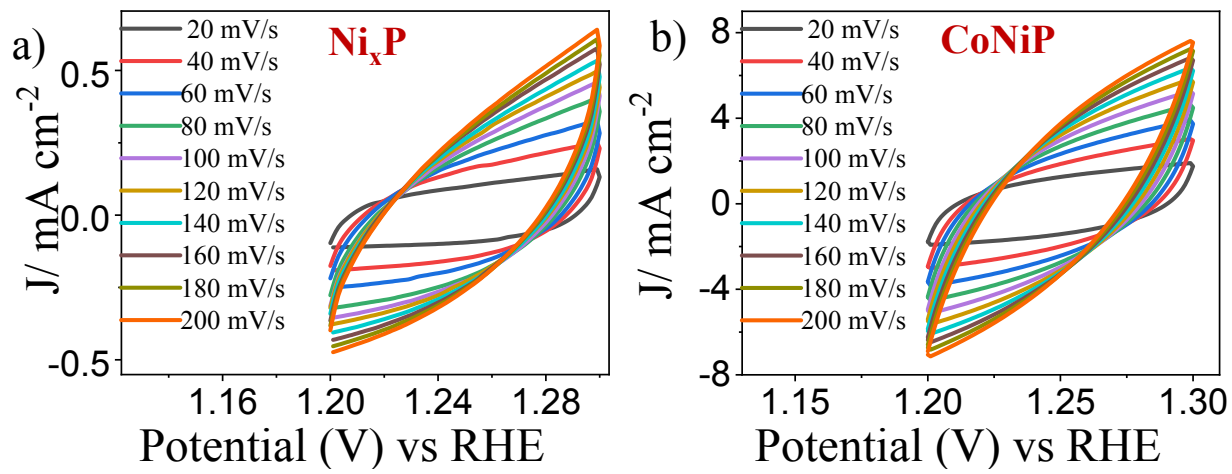

Fig. S4: Cyclic voltammograms of a) NiP and b) CoNiP@NF electrocatalysts. 

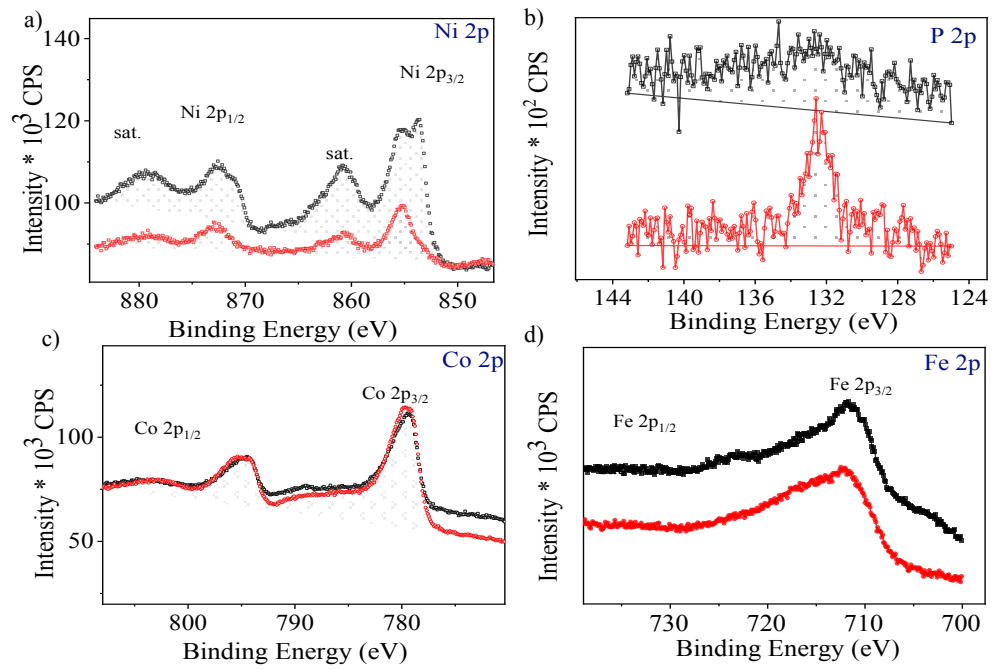

Fig. S5: The core level XPS spectra (a) Ni 2p, (b) P 2p, (c) Co 2p and (d) Fe 2p after HER and OER processes.

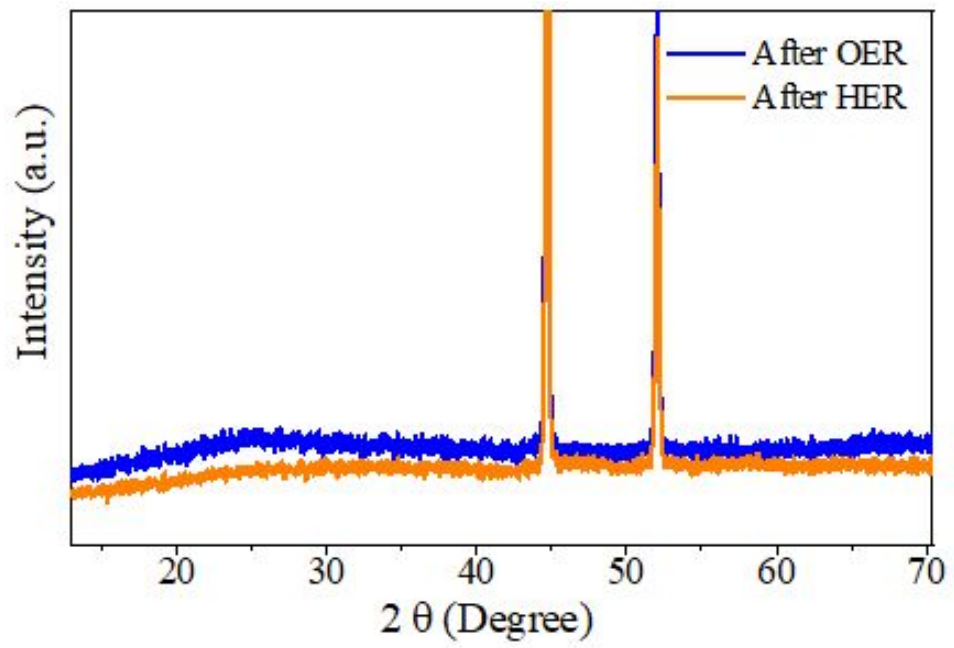

Fig. S6: The XRD patterns of Fe-CoNiP after HER and OER electrolysis.

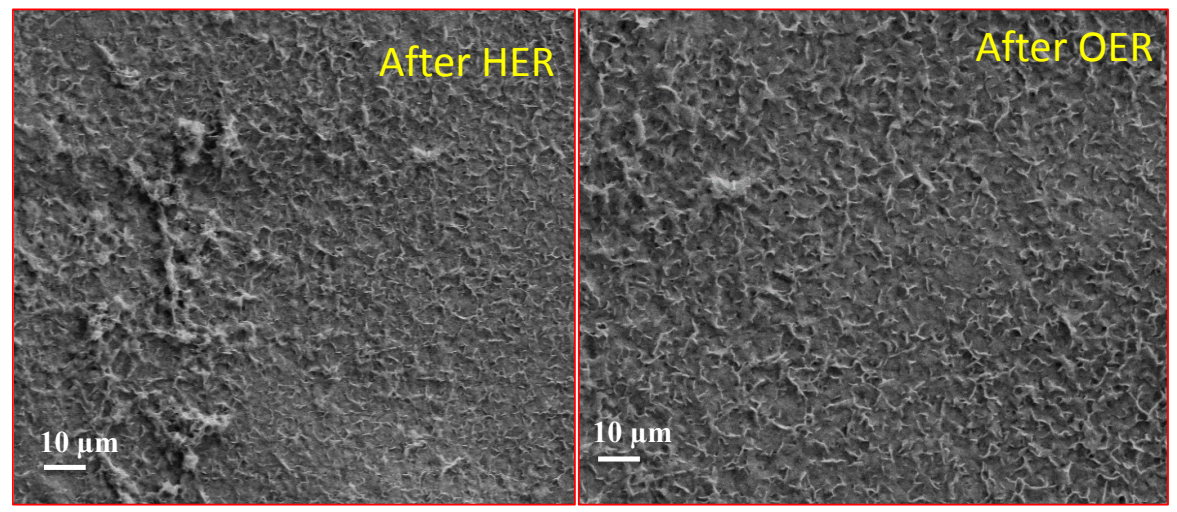

Fig. S7: The SEM images of Fe-CoNiP after HER and OER electrolysis. 

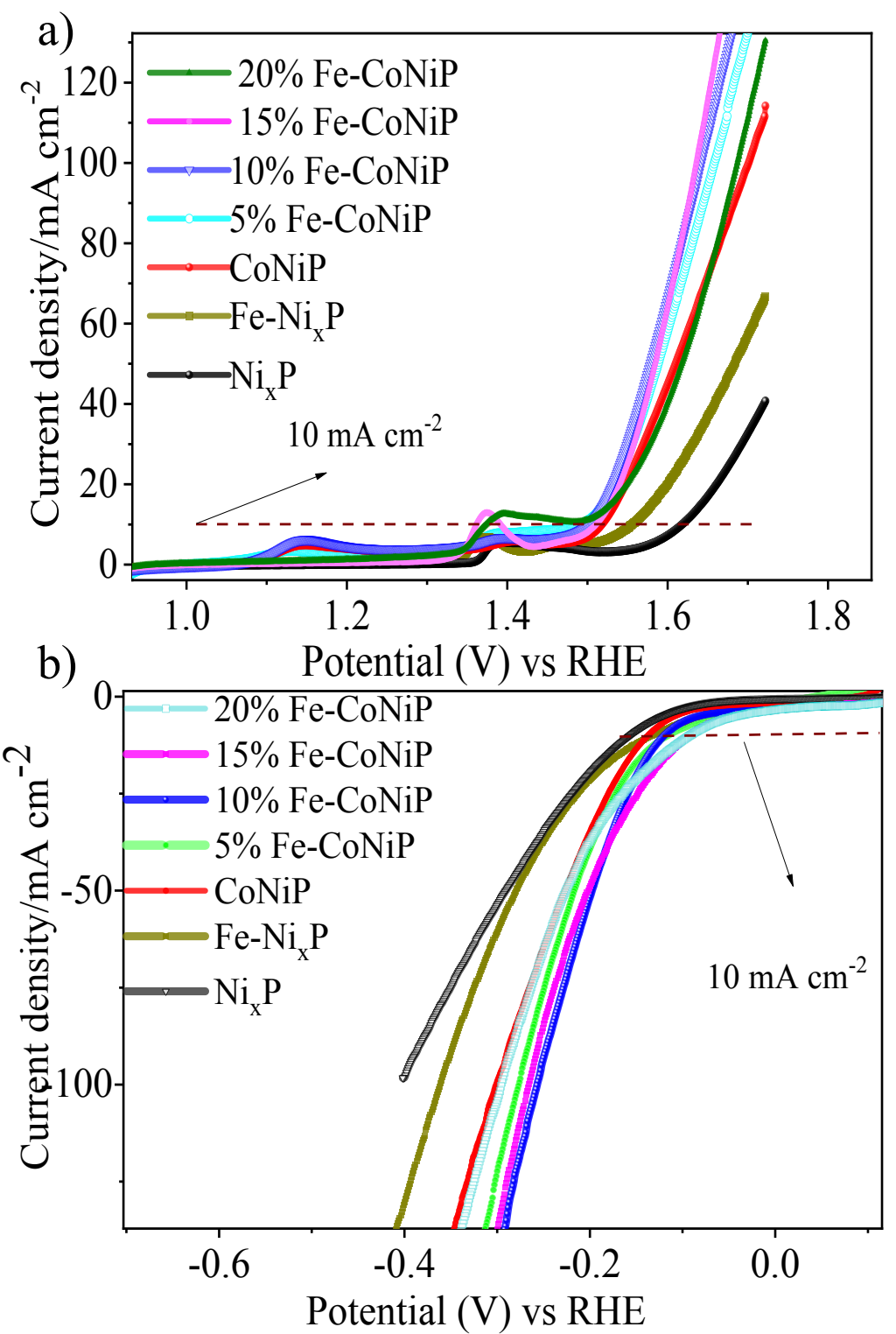

Fig. S8: LSV Polarization a) OER b) HER of various electrocatalysts 


\section{References}

1. Yuan, C.-Z.; Zhong, S.-L.; Jiang, Y.-F.; Yang, Z. K.; Zhao, Z.-W.; Zhao, S.-J.; Jiang, N.; Xu, A.-W., Direct Growth of Cobalt-Rich Cobalt Phosphide Catalysts on Cobalt Foil: An Efficient and Self-Supported Bifunctional Electrode for Overall Water Splitting in Alkaline Media. Journal of Materials Chemistry A 2017, 5, 10561-10566.

2. Jiao, L.; Zhou, Y.-X.; Jiang, H.-L., Metal-Organic Framework-Based Cop/Reduced Graphene Oxide: High-Performance Bifunctional Electrocatalyst for Overall Water Splitting. Chemical Science 2016, 7, 1690-1695.

3. Li, W.; Zhang, S.; Fan, Q.; Zhang, F.; Xu, S., Hierarchically Scaffolded Cop/Cop 2 Nanoparticles: Controllable Synthesis and Their Application as a Well-Matched Bifunctional Electrocatalyst for Overall Water Splitting. Nanoscale 2017, 9, 5677-5685.

4. Yu, J.; Cheng, G.; Luo, W., Ternary Nickel-Iron Sulfide Microflowers as a Robust Electrocatalyst for Bifunctional Water Splitting. Journal of Materials Chemistry A 2017, $5,15838-15844$.

5. Wan, H.; Lv, M.; Liu, X.; Chen, G.; Zhang, N.; Cao, Y.; Wang, H.; Ma, R.; Qiu, G., Activating Hematite Nanoplates Via Partial Reduction for Electrocatalytic Oxygen Reduction Reaction. ACS Sustainable Chemistry \& Engineering 2019, 7, 11841-11849.

6. Chunduri, A.; Gupta, S.; Bapat, O.; Bhide, A.; Fernandes, R.; Patel, M.; Bambole, V.; Miotello, A.; Patel, N., A Unique Amorphous Cobalt-Phosphide-Boride Bifunctional Electrocatalyst for Enhanced Alkaline Water-Splitting. Applied Catalysis B: Environmental 2019, 259, 118051.

7. Li, Y.; Wang, H.; Li, Y.; Wang, Q.; Li, D.; Wang, R.; He, B.; Gong, Y., 2D MetalOrganic-Framework Array-Derived Hierarchical Network Architecture of Cobalt Oxide Flakes with Tunable Oxygen Vacancies Towards Efficient Oxygen Evolution Reaction. Journal of Catalysis 2018, 364, 48-56.

8. Wang, P. c.; Wan, L.; Lin, Y. Q.; Wang, B. g., NiFe Hydroxide Supported on Hierarchically Porous Nickel Mesh as a High-Performance Bifunctional Electrocatalyst for Water Splitting at Large Current Density. ChemSusChem 2019, 12, 4038-4045.

9. Li, F. L.; Shao, Q.; Huang, X.; Lang, J. P., Nanoscale Trimetallic Metal-Organic Frameworks Enable Efficient Oxygen Evolution Electrocatalysis. Angewandte Chemie 2018, 130, 1906-1910.

10. Luo, J.; Im, J.-H.; Mayer, M. T.; Schreier, M.; Nazeeruddin, M. K.; Park, N.-G.; Tilley, S. D.; Fan, H. J.; Grätzel, M., Water Photolysis at 12.3\% Efficiency Via Perovskite Photovoltaics and Earth-Abundant Catalysts. Science 2014, 345, 1593-1596.

11. Li, Y.; Zhang, H.; Jiang, M.; Zhang, Q.; He, P.; Sun, X., 3D Self-Supported Fe-Doped $\mathrm{Ni}_{2} \mathrm{P}$ Nanosheet Arrays as Bifunctional Catalysts for Overall Water Splitting. Advanced Functional Materials 2017, 27, 1702513. 